CARPATHIAN J. MATH.

Volume 37 (2021), No. 2,

Pages $265-272$
Online version at https : //www . carpathian. cunbm . utcluj. ro/

Print Edition: ISSN 1584 - 2851; Online Edition: ISSN 1843 - 4401

DOI: https://doi.org/10.37193/CJM.2021.02.12

Dedicated to Prof. Ioan A. Rus on the occasion of his $85^{\text {th }}$ anniversary

\title{
Some remarks on the metrizability of some metric-like structures
}

\author{
Sumit SOM ${ }^{1}$, AdriAn Petruşel $^{2,3}$ and LAKShMi KANTA DeY ${ }^{4}$
}

\begin{abstract}
The main purpose of this article is to provide alternative proofs of the metrizability of metric-like spaces like $b$-metric spaces, $\mathcal{F}$-metric spaces, and $\theta$-metric spaces. We improve upon the metrizability result of An et al. [Topology Appl. 185-186 (2015)] for $b$-metric spaces. Moreover, we provide two shorter proofs of the metrizability of $\mathcal{F}$-metric spaces, recently introduced by Jleli and Samet. Furthermore, we give a partial answer to an open problem regarding the openness of $\mathcal{F}$-open balls in $\mathcal{F}$-metric spaces. Finally, we give an alternative proof of the metrizability of $\theta$-metric spaces.
\end{abstract}

\section{Metrizability OF $b$-Metric SPACES}

In 1993, Czerwik [9] defined the notion of $b$-metric as a generalization of the metric functional, by modifying the triangle inequality axiom. Further, in 1998, Czerwik [10] modified again the notion, by considering instead of the coefficient 2 in the right hand side of the relation an arbitrary coefficient $K \geq 1$. Surprisingly, in 1998, Aimar et al. [1] proved the metrizability of such spaces. In this sequel, intendant readers can see $[8,15]$ more results about $b$-metric spaces. In 2010, Khamsi and Hussain [13] reconsidered the concept of a $b$-metric under the name metric-type spaces, where they had considered the coefficient to be $K>0$. To avoid confusion, the metric-type in the sense of Khamsi and Hussain [13] will be called $b$-metric in this short note. For related results and applications in $b$-metric spaces see also [4-6] and [17]. Before going further, we like to recall the definition of a $b$-metric space from [13] as follows:

Definition 1.1. [13, Definition 6.] Let $X$ be a non-empty set and $K>0$. A distance function $D: X \times X \rightarrow[0, \infty)$ is said to be a $b$-metric on $X$ if it satisfies the following conditions:

(i) $D(x, y)=0 \Longleftrightarrow x=y$ for all $(x, y) \in X \times X$;

(ii) $D(x, y)=D(y, x)$ for all $(x, y) \in X \times X$;

(iii) $D(x, z) \leq K[D(x, y)+D(y, z)]$ for all $x, y, z \in X$.

Then the triple $(X, D, K)$ is called a $b$-metric space. If we take $K=1$, then $X$ becomes a metric space. So, $b$-metric spaces are more general than the standard metric spaces. In 2015, An et al. [2] presented a proof for the metrizability of $b$-metric spaces with coefficient $K>0$. However, they proved the metrizability result on an assumption that the distance function is continuous in one variable. We will state first the main theorem and its corollary due to An et al.

Received: 22.10.2020. In revised form: 16.04.2021. Accepted: 23.04.2021

2010 Mathematics Subject Classification. 54E35; 54H99; 47H10; 54H25.

Key words and phrases. $b$-metric space, $\mathcal{F}$-metric space, $\theta$-metric space, metrizability.

Corresponding author: A. Petruşel; petrusel@math.ubbcluj.ro 
Theorem 1.1. [2, Theorem 3.15.] Let $(X, D, K)$ be a b-metric space. If $D$ is continuous in one variable then every open cover of $X$ has an open refinement which is both locally finite and $\sigma$-discrete.

Corollary 1.1. [2, Corollary 3.17.] Let $(X, D, K)$ be a b-metric space. If $D$ is continuous in one variable then $X$ is metrizable.

One of the main motivation of this short note is to give a simple proof of the metrizability of $b$-metric spaces with coefficient $K>0$ without considering any continuity assumption. We use metrization theorem due to Niemytski and Wilson in our proof. Before proceeding to our metrizability result, we like to recall the metrization theorem due to Niemytski and Wilson as follows:

Theorem 1.2. [11, Page 137.] Let $X$ be a topological space and $F: X \times X \rightarrow[0, \infty)$ be a distance function on $X$. If the distance function $F$ satisfies

(i) $F(x, y)=0 \Longleftrightarrow x=y$ for all $(x, y) \in X \times X$;

(ii) $F(x, y)=F(y, x)$ for all $(x, y) \in X \times X$

and one of the following conditions:

(iii-A) Given a point $a \in X$ and a number $\varepsilon>0$, there exists $\phi(a, \varepsilon)>0$ such that if $F(a, b)<$ $\phi(a, \varepsilon)$ and $F(b, c)<\phi(a, \varepsilon)$ then $F(a, c)<\varepsilon ;$

(iii-B) if $a \in X$ and $\left\{a_{n}\right\}_{n \in \mathbb{N}},\left\{b_{n}\right\}_{n \in \mathbb{N}}$ are two sequences in $X$ such that $F\left(a_{n}, a\right) \rightarrow 0$ and $F\left(a_{n}, b_{n}\right) \rightarrow 0$ as $n \rightarrow \infty$ then $F\left(b_{n}, a\right) \rightarrow 0$ as $n \rightarrow \infty$;

(iii-C) for each point $a \in X$ and positive number $k$, there is a positive number $r$ such that if $b \in X$ for which $F(a, b) \geq k$, and $c$ is any point then $F(a, c)+F(b, c) \geq r$,

then the topological space $X$ is metrizable.

In [16], Niemytski had considered the condition (iii-A) of Theorem 1.2 and showed that a space with a distance function satisfying the conditions (i), (ii) and (iii-A) is metrizable. This condition (iii-A), given by Niemytski in [16], is known as the local axiom of the triangle. In [16], Niemytski also showed that the conditions (iii-A) and (iii-B) of Theorem 1.2 are equivalent. Also it can be easily seen that conditions (iii-C) and (iii-A) of Theorem 1.2 are also equivalent. In [19], Wilson considered condition (iii-C) and showed that a space with a distance function satisfying the conditions (i), (ii) and (iii-C) is metrizable.

Theorem 1.3. Let $(X, D, K), K>0$ be a b-metric space. Then $X$ is metrizable.

Proof. Let $(X, D, K)$ be a $b$-metric space. By the definition of a $b$-metric space, the distance function $D: X \times X \rightarrow[0, \infty)$ on $X$ satisfies the first two conditions of Niemytski and Wilson's metrization result, i.e,

(i) $D(x, y)=0 \Longleftrightarrow x=y$ for all $(x, y) \in X \times X$;

(ii) $D(x, y)=D(y, x)$ for all $(x, y) \in X \times X$.

Now we prove the third condition, i.e., the "locally regular" condition and for that, we prove the condition (iii-C) of Theorem 1.2. Let $a \in X$ and $t$ be a positive real number. Assume that $b \in X$ such that $D(a, b) \geq t$. If $c$ is any point in $X$ then by the definition of a $b$-metric space we have,

$$
D(a, b) \leq K(D(a, c)+D(c, b)) \Longrightarrow(D(a, c)+D(c, b)) \geq \frac{t}{K}=r>0 .
$$

This shows that the distance function $D: X \times X \rightarrow[0, \infty)$ of a $b$-metric space satisfies the locally regular condition. Similarly conditions (iii-A) and (iii-B) of Theorem 1.2 are easily satisfied by any $b$-metric. Consequently, by Niemytski and Wilson's metrization theorem we can conclude that the $b$-metric space $X$ is metrizable. 
Remark 1.1. The above metrizability result is superior, in some sense, to the ones in $[1,2,8]$.

Remark 1.2. From Theorem 1.3, we can conclude that if $(X, D, K), K>0$ is a $b$-metric space, then there exists a metric $d: X \times X \rightarrow[0, \infty)$ on $X$ such that $X$ is metrizable with respect to the metric $d$. Thus, the topological properties of $b$-metric spaces discussed in [13, Proposition 2, Proposition 3] are equivalent to those of the standard metric spaces.

\section{MetrizABiLity OF $\mathcal{F}$-METRIC SPACES}

Recently, Jleli and Samet [12] proposed a new generalization of the usual notion of metric spaces. By means of a certain class of functions, the authors defined the notion of an $\mathcal{F}$-metric space. Let us first recall the definition of such spaces. Let $\mathcal{F}$ denote the class of functions $f:(0, \infty) \rightarrow \mathbb{R}$ which satisfy the following conditions:

$\left(\mathcal{F}_{1}\right) f$ is non-decreasing, i.e., $0<s<t \Rightarrow f(s) \leq f(t)$.

$\left(\mathcal{F}_{2}\right)$ For every sequence $\left\{t_{n}\right\}_{n \in \mathbb{N}} \subseteq(0,+\infty)$, we have

$$
\lim _{n \rightarrow+\infty} t_{n}=0 \Longleftrightarrow \lim _{n \rightarrow+\infty} f\left(t_{n}\right)=-\infty \text {. }
$$

The definition of an $\mathcal{F}$-metric space has been introduced as follows.

Definition 2.2. [12, Definition 2.1.] Let $X$ be a non-empty set and $D: X \times X \rightarrow[0, \infty)$ be a given mapping. Suppose there exists $(f, \alpha) \in \mathcal{F} \times[0, \infty)$ such that:

(D1) $D(x, y)=0 \Longleftrightarrow x=y$ for all $(x, y) \in X \times X$.

(D2) $D(x, y)=D(y, x)$ for all $(x, y) \in X \times X$.

(D3) For every $(x, y) \in X \times X$, for each $N \in \mathbb{N}, N \geq 2$ and for every $\left(u_{i}\right)_{i=1}^{N} \subseteq X$ with $\left(u_{1}, u_{N}\right)=(x, y)$, we have

$$
D(x, y)>0 \Longrightarrow f(D(x, y)) \leq f\left(\sum_{i=1}^{N-1} D\left(u_{i}, u_{i+1}\right)\right)+\alpha .
$$

Then $D$ is said to be an $\mathcal{F}$-metric on $X$ and the pair $(X, D)$ is said to be an $\mathcal{F}$-metric space. Hence, the class of all $\mathcal{F}$-metric spaces contains the class of all metric spaces, obtained for $f(t)=$ lnt and $\alpha=0$. The following definitions and propositions from [12] will be needed.

Definition 2.3. [12, Definition 4.1.] Let $(X, D)$ be an $\mathcal{F}$-metric space. A subset $C$ of $X$ is said to be $\mathcal{F}$-open if for every $x \in C$, there is some $r>0$ such that $B(x, r) \subset C$, where $B(x, r):=\{y \in X: D(y, x)<r\}$. We say that a subset $C$ of $X$ is $\mathcal{F}$-closed if $X \backslash C$ is $\mathcal{F}$-open. The family of all $\mathcal{F}$-open subsets of $X$ is denoted by $\tau_{\mathcal{F}}$.

Definition 2.4. [12, Definition 4.3.] Let $(X, D)$ be an $\mathcal{F}$-metric space. Let $\left\{x_{n}\right\}_{n \in \mathbb{N}}$ be a sequence in $X$. We say that $\left\{x_{n}\right\}_{n \in \mathbb{N}}$ is $\mathcal{F}$-convergent to $x \in X$ if $\left\{x_{n}\right\}_{n \in \mathbb{N}}$ is convergent to $x \in X$ with respect to the topology $\tau_{\mathcal{F}}$.

Proposition 2.1. [12, Proposition 4.4.] Let $(X, D)$ be an $\mathcal{F}$-metric space. Then, for any nonempty subset $A$ of $X$, the following implication holds $x \in \bar{A}, r>0 \Longrightarrow B(x, r) \cap A \neq \emptyset$.

Proposition 2.2. [12, Proposition 4.5.] Let $(X, D)$ be an $\mathcal{F}$-metric space. Let $\left\{x_{n}\right\}_{n \in \mathbb{N}}$ be a sequence in $X$ and $x \in X$. Then the following are equivalent:

(i) $\left\{x_{n}\right\}_{n \in \mathbb{N}}$ is $\mathcal{F}$-convergent to $x$.

(ii) $D\left(x_{n}, x\right) \rightarrow 0$ as $n \rightarrow \infty$.

Proposition 2.3. [12, Proposition 4.6.] Let $(X, D)$ be an $\mathcal{F}$-metric space and $\left\{x_{n}\right\}_{n \in \mathbb{N}}$ be a sequence in $X$. Then

$$
(x, y) \in X \times X, \lim _{n \rightarrow \infty} D\left(x_{n}, x\right)=\lim _{n \rightarrow \infty} D\left(x_{n}, y\right)=0 \Longrightarrow x=y .
$$


Very recently Som et al. [18] proved that this newly defined structure is metrizable by using the definition of metrizability. However, their proof is technical and a bit lengthy. In this short note, we give two alternative proofs of metrizability of this structure using Chittenden's metrization theorem [7] and metrization theorem due to Niemytski and Wilson (discussed in Theorem 1.2). It may be noted that these proofs are very simple. Before proceeding to the metrizability result for $\mathcal{F}$-metric spaces, we recall the metrization result due to Chittenden [7].

Theorem 2.4. [7] Let $X$ be a topological space and $F: X \times X \rightarrow[0, \infty)$ be a distance function on $X$. If the distance function $F$ satisfies the following conditions:

(i) $F(x, y)=0 \Longleftrightarrow x=y$ for all $(x, y) \in X \times X$;

(ii) $F(x, y)=F(y, x)$, for all $(x, y) \in X \times X$;

(iii) (Uniformly regular) For every $\varepsilon>0$ there exists $\phi(\varepsilon)>0$ such that for all $x, y, z \in X$, $F(x, y)<\phi(\varepsilon)$ and $F(y, z)<\phi(\varepsilon)$ imply $F(x, z)<\varepsilon$,

then the topological space $X$ is metrizable.

Now in the upcoming theorem, we present, by two different approaches, two short proofs of the metrizability of $\mathcal{F}$-metric spaces. The first approach is by using Chittenden's metrization theorem, while the second one is by using Niemytski and Wilson's metrization theorem.

Theorem 2.5. Let $(X, D)$ be an $\mathcal{F}$-metric space with $(f, \alpha) \in \mathcal{F} \times[0, \infty)$. Then $X$ is metrizable.

Proof. Approach I. Let $X$ be an $\mathcal{F}$-metric space with $(f, \alpha) \in \mathcal{F} \times[0, \infty)$. By the definition of an $\mathcal{F}$-metric space, the distance function $D: X \times X \rightarrow[0, \infty)$ satisfies the first two conditions of Chittenden's metrization result, i.e,

(i) $D(x, y)=0 \Longleftrightarrow x=y$ for all $(x, y) \in X \times X$.

(ii) $D(x, y)=D(y, x)$ for all $(x, y) \in X \times X$.

Now we prove the third condition, i.e., the "uniformly regular" condition. Let $\varepsilon>0$ and $x, y, z \in X$. If $x=z$, then $D(x, z)=0$. So in this case $\phi(\varepsilon)=c$ where $c$ is any positive real number will serve the purpose. Let $x \neq z$. Then $D(x, z)>0$. So, by the definition of an $\mathcal{F}$-metric space we have

$$
f(D(x, z)) \leq f(D(x, y)+D(y, z))+\alpha .
$$

By the $\mathcal{F}_{2}$ condition, for $(f(\varepsilon)-\alpha) \in \mathbb{R}$ there exists $\delta>0$ such that $0<t<\delta \Longrightarrow f(t)<$ $f(\varepsilon)-\alpha$. Let us choose $\phi(\varepsilon)=\frac{\delta}{2}$. If $D(x, y)<\frac{\delta}{2}$ and $D(y, z)<\frac{\delta}{2}$ then $D(x, y)+D(y, z)<\delta$. Thus, by (2.1), the following implication holds $f(D(x, z))<f(\varepsilon) \Longrightarrow D(x, z)<\varepsilon$. This shows that the distance function $D$ of an $\mathcal{F}$-metric space satisfies the uniformly regular condition. Consequently, by Chittenden's metrization result we can conclude that the $\mathcal{F}$-metric space $X$ is metrizable.

Approach II. In this part we show that any $\mathcal{F}$-metric $D: X \times X \rightarrow[0, \infty)$ satisfies condition (iii-B) of Theorem 1.2. The interested reader can also check that, the $\mathcal{F}$-metric $D: X \times X \rightarrow[0, \infty)$ satisfies condition (iii-A) of Theorem 1.2 , by proceeding similarly as the proof of "uniformly regular" condition in Theorem 2.5 under approach I. Let $a \in X$ and $\left\{a_{n}\right\}_{n \in \mathbb{N}},\left\{b_{n}\right\}_{n \in \mathbb{N}}$ are two sequences in $X$ such that $D\left(a_{n}, a\right) \rightarrow 0$ and $D\left(a_{n}, b_{n}\right) \rightarrow 0$ as $n \rightarrow \infty$. Let $\varepsilon>0$. By $\mathcal{F}_{2}$ condition, for $(f(\varepsilon)-\alpha) \in \mathbb{R}$ there exists $\delta>0$ such that $0<t<\delta \Longrightarrow f(t)<f(\varepsilon)-\alpha$. For $\frac{\delta}{2}>0$, there exists $k_{1}, k_{2} \in \mathbb{N}$ such that

$$
D\left(a_{n}, a\right)<\frac{\delta}{2}, \forall n \geq k_{1} \text { and } D\left(a_{n}, b_{n}\right)<\frac{\delta}{2}, \forall n \geq k_{2} .
$$

Now if $n \geq \max \left\{k_{1}, k_{2}\right\}$ and $a \neq b_{n}$, then by the definition of an $\mathcal{F}$-metric space, we have

$$
f\left(D\left(a, b_{n}\right)\right) \leq f\left(D\left(a, a_{n}\right)+D\left(a_{n}, b_{n}\right)\right)+\alpha \Longrightarrow f\left(D\left(a, b_{n}\right)\right)<f(\varepsilon) \Longrightarrow D\left(a, b_{n}\right)<\varepsilon .
$$


This shows that $D\left(b_{n}, a\right) \rightarrow 0$ as $n \rightarrow \infty$.

Thus, by the metrization criterion due to Niemytski and Wilson, we can conclude that the $\mathcal{F}$-metric space $X$ is metrizable.

Remark 2.3. From Theorem 2.5 we can conclude that if $(X, D)$ be an $\mathcal{F}$-metric space then there exists a metric $d: X \times X \rightarrow[0, \infty)$ on $X$ such that $X$ is metrizable with respect to the metric $d$. So, the topological properties of $\mathcal{F}$-metric spaces discussed in Proposition 2.1-2.3 are equivalent to those of the standard metric counterparts.

In [12], Jleli and Samet obtained a few interesting results regarding the topology of $\mathcal{F}$ metric spaces. This study was further continued by Bera et al. [3], where they posed the following interesting open problem.

Open question. Is every open ball an $\mathcal{F}$-open set in $\mathcal{F}$-metric spaces?

Next, we give a partial answer to this problem by finding an upper bound of the radius of the open balls for which the open balls become open. We also acquire a necessary and sufficient condition under which an open ball in $\mathcal{F}$-metric spaces becomes $\mathcal{F}$-open.

Theorem 2.6. Let $X$ be an $\mathcal{F}$-metric space with $(f, \alpha) \in \mathcal{F} \times[0, \infty)$ and $B_{D}(x, r)=\{y \in X$ : $D(y, x)<r\}$ denotes an $\mathcal{F}$-open ball with center at $x$ and radius $r>0$. Then $B_{D}(x, r)$ is $\mathcal{F}$-open if and only if $B_{D}(x, r)$ is open with respect to the metric $d$ defined by

$$
d(x, y)=\inf \left\{\sum_{i=1}^{N-1} D\left(u_{i}, u_{i+1}\right): N \in \mathbb{N}, N \geq 2,\left\{u_{i}\right\}_{i=1}^{N} \subseteq X \text { with }\left(u_{1}, u_{N}\right)=(x, y)\right\} .
$$

Proof. The proof can be easily done using [18, Theorem 2.1.], so it is omitted.

Let $X$ be an $\mathcal{F}$-metric space with $(f, \alpha) \in \mathcal{F} \times[0, \infty)$. Let $r>0$. By the condition $\left(\mathcal{F}_{2}\right)$, for $(f(r)-\alpha) \in \mathbb{R}$, there exists $\delta>0$ such that $0<t<\delta \Longrightarrow f(t)<f(r)-\alpha$. This quantity $\delta$ depends on $r$ and $\alpha$. We will denote this $\delta$ by $W(r, \alpha)$. In our next theorem, we present a sufficient condition under which the open ball $B_{D}(x, r)$ is $\mathcal{F}$-open.

Theorem 2.7. Let $X$ be an $\mathcal{F}$-metric space with $(f, \alpha) \in \mathcal{F} \times[0, \infty)$ and $B_{D}(x, r)=\{y \in X$ : $D(y, x)<r\}$ denotes an $\mathcal{F}$-open ball with center at $x$ and radius $r>0$. If $r<\frac{W(r, \alpha)}{2}$, then $B_{D}(x, r)$ is $\mathcal{F}$-open.

Proof. We will prove that $B_{D}(x, r)^{c}=\{y \in X: D(y, x) \geq r\}$ is closed with respect to the metric $d$ defined in equation (2.2). Let $z \in \overline{B_{D}(x, r)^{c}}$. Then there exists a sequence $\left\{z_{n}\right\}_{n \in \mathbb{N}} \subset B_{D}(x, r)^{c}$ such that $z_{n} \rightarrow z$ as $n \rightarrow \infty$ with respect to the metric $d$. So $D\left(z_{n}, x\right) \geq r$ for all $n \in \mathbb{N}$ and $d\left(z_{n}, z\right) \rightarrow 0$ as $n \rightarrow \infty$. Let $z \notin B_{D}(x, r)^{c}$. Then $z \in B_{D}(x, r)$. So $D(z, x)<r$. This implies $d(z, x) \leq D(z, x)<r$. Take $\frac{W(r, \alpha)}{4}>0$. So there exists $K \in \mathbb{N}$ such that $d\left(z_{n}, z\right)<\frac{W(r, \alpha)}{4}$ for all $n \geq K$. Now we take $z_{n} \in B_{D}(x, r)^{c}$ with $n \geq K$. So, $D\left(z_{n}, x\right)>0$. From the relation between the $\mathcal{F}$-metric $D$ and the metric $d$ defined in equation (2.2) we have

$$
\begin{aligned}
f\left(D\left(z_{n}, x\right)\right) & \leq f\left(d\left(z_{n}, x\right)+\frac{W(r, \alpha)}{4}\right)+\alpha \\
& \leq f\left(d\left(z_{n}, z\right)+d(z, x)+\frac{W(r, \alpha)}{4}\right)+\alpha \\
& \leq f\left(d\left(z_{n}, z\right)+r+\frac{W(r, \alpha)}{4}\right)+\alpha \\
& \leq f\left(d\left(z_{n}, z\right)+\frac{W(r, \alpha)}{2}+\frac{W(r, \alpha)}{4}\right)+\alpha<f(r) \\
& \Rightarrow D\left(z_{n}, x\right)<r .
\end{aligned}
$$


This is a contradiction, since $z_{n} \in B_{D}(x, r)^{c}$. Thus $z \in B_{D}(x, r)^{c}$. This shows that $B_{D}(x, r)^{c}$ is closed with respect to the metric $d$. So, $B_{D}(x, r)$ is open with respect to $d$. So, by [18, Theorem 2.1.], we can conclude that $B_{D}(x, r)$ is $\mathcal{F}$-open.

\section{Metrizability of $\theta$-Metric SPACES}

In 2013, Khojasteh et al. [14] introduced the notion of a $\theta$-metric space by using the concept of a $B$-action on the set $[0, \infty) \times[0, \infty)$. Before proceeding to the definition of $\theta$-metric space, we recall the definition of a $B$-action (see [14]), as follows:

Definition 3.5. [14, Definition 4.] Let $\theta:[0, \infty) \times[0, \infty) \rightarrow[0, \infty)$ be a continuous mapping with respect to each variable. Let $\operatorname{Im}(\theta)=\{\theta(s, t): s, t \geq 0\}$. Then $\theta$ is called a $B$-action if and only if the following conditions are satisfied:

(i) $\theta(0,0)=0$ and $\theta(s, t)=\theta(t, s)$ for all $s, t \geq 0$;

(ii) $\theta(x, y)<\theta(s, t)$ if either $x \leq s, y<t$ or $x<s, y \leq t$;

(iii) For each $m \in \operatorname{Im}(\theta)$ and for each $t \in[0, m]$, there exists $s \in[0, m]$ such that $\theta(s, t)=m$

(iv) $\theta(s, 0) \leq s$ for all $s>0$.

Authors denoted the collection of all such $B$-actions by $Y$. Now, we will recall (see [14]) the definition of a $\theta$-metric space, as follows.

Definition 3.6. [14, Definition 11.] Let $X$ be a non-empty set. A distance function $d$ : $X \times X \rightarrow[0, \infty)$ is said to be a $\theta$-metric on $X$ with respect to a $B$-action $\theta \in Y$ if the following conditions are satisfied:

(i) $d(x, y)=0 \Longleftrightarrow x=y$ for all $(x, y) \in X \times X$;

(ii) $d(x, y)=d(y, x)$ for all $(x, y) \in X \times X$;

(iii) $d(x, z) \leq \theta(d(x, y), d(y, z))$ for all $x, y, z \in X$.

The triple $(X, d, \theta)$ is called a $\theta$-metric space. If we take $\theta(s, t)=s+t, s, t \geq 0$ then $\theta$-metric space reduce to metric space. In the same paper, Khojasteh et al. [14] also developed some topological structure induced by the $\theta$-metric and concluded that it is a metrizable topological space. However their proof of metrizability relies on the prior knowledge of the uniformity of an uniform space $X$. In our paper, we prove the metrizability of $\theta$-metric spaces by using the well-known Niemytski and Wilson's metrization theorem.

Theorem 3.8. Let $(X, d, \theta)$ be a $\theta$-metric space, where $\theta$ is a $B$-action on $[0, \infty) \times[0, \infty)$. Then $X$ is metrizable.

Proof. Throughout this proof, we will use the standard norm on the set $[0, \infty) \times[0, \infty)$ as $\|(x, y)\|=\sqrt{x^{2}+y^{2}}, x, y \geq 0$. First of all, we show that the $B$-action $\theta$ is continuous at the point $(0,0)$. Suppose that $\left\{\left(s_{n}, t_{n}\right)\right\}_{n \in \mathbb{N}}$ is a sequence in $[0, \infty) \times[0, \infty)$, such that $\left(s_{n}, t_{n}\right) \rightarrow(0,0)$ as $n \rightarrow \infty$. This implies $s_{n} \rightarrow 0$ and $t_{n} \rightarrow 0$ as $n \rightarrow \infty$ in the standard norm in $[0, \infty) \times[0, \infty)$. Now, as the $B$-action $\theta$ is continuous in both of the variables, we get that $\theta\left(s_{n}, t_{n}\right) \rightarrow \theta(0,0)=0$ as $n \rightarrow \infty$. This shows that the $B$-action $\theta$ is continuous at the point $(0,0)$. Now we prove that $X$ is metrizable. By the definition of a $\theta$-metric space, the distance function $d: X \times X \rightarrow[0, \infty)$ on $X$ satisfies the first two conditions of Niemytski and Wilson's metrization result, i.e,

(i) $d(x, y)=0 \Longleftrightarrow x=y$ for all $(x, y) \in X \times X$;

(ii) $d(x, y)=d(y, x)$ for all $(x, y) \in X \times X$.

We show that any $\theta$-metric $d: X \times X \rightarrow[0, \infty)$ satisfies the condition (iii-B) and (iii-C) of Theorem 1.2. It is easy to check that the $\theta$-metric $d: X \times X \rightarrow[0, \infty)$ also satisfies the 
condition (iii-A) of Theorem 1.2. Let $a \in X$ and $\left\{a_{n}\right\}_{n \in \mathbb{N}},\left\{b_{n}\right\}_{n \in \mathbb{N}}$ are two sequences in $X$ such that $d\left(a_{n}, a\right) \rightarrow 0$ and $d\left(a_{n}, b_{n}\right) \rightarrow 0$ as $n \rightarrow \infty$. We show that $d\left(b_{n}, a\right) \rightarrow 0$ as $n \rightarrow \infty$. Now $\left(d\left(a_{n}, a\right), d\left(a_{n}, b_{n}\right)\right) \rightarrow(0,0)$ as $n \rightarrow \infty$ in the standard norm on $[0, \infty) \times[0, \infty)$. As the $B$-action $\theta$ is continuous at the point $(0,0)$, we get that $\theta\left(d\left(a_{n}, a\right), d\left(a_{n}, b_{n}\right)\right) \rightarrow \theta(0,0)=0$ as $n \rightarrow \infty$. Now from the definition of $\theta$-metric space we have,

$$
d\left(a, b_{n}\right) \leq \theta\left(d\left(a_{n}, a\right), d\left(a_{n}, b_{n}\right)\right) \Longrightarrow d\left(a, b_{n}\right) \rightarrow 0 \text { as } n \rightarrow \infty .
$$

So the $\theta$-metric $d: X \times X \rightarrow[0, \infty)$ satisfies the condition (iii-B) of Theorem 1.2. Now we check for condition (iii-C). Let $a \in X$ and $k>0$. Let $b \in X$ such that $d(a, b) \geq k$. As the $B$-action $\theta$ is continuous at the point $(0,0)$, so for $k>0$ there exists $\delta>0$ such that

$$
\theta(x, y)<k \text { whenever }(x, y) \in B((0,0), \delta) \bigcap([0, \infty) \times[0, \infty)) .
$$

Here $B((0,0), \delta)$ denotes the open ball centered at $(0,0)$ and radius $\delta$ in the standard norm, i.e, $B((0,0), \delta)=\left\{(x, y) \in \mathbb{R}^{2}:\|(x, y)\|<\delta\right\}$. Let $c \in X$. From the definition of $\theta$-metric space we have

$$
\begin{gathered}
d(a, b) \leq \theta(d(a, c), d(c, b)) \Longrightarrow \theta(d(a, c), d(c, b)) \geq k \Longrightarrow \\
(d(a, c), d(c, b)) \notin B((0,0), \delta) \bigcap([0, \infty) \times[0, \infty)) \Longrightarrow \\
d^{2}(a, c)+d^{2}(c, b) \geq \delta^{2},
\end{gathered}
$$

as $(d(a, c), d(c, b)) \in[0, \infty) \times[0, \infty)$, so, $(d(a, c), d(c, b)) \notin B((0,0), \delta)$.

So, either $d(a, c) \geq \frac{\delta}{\sqrt{2}}$ or $d(c, b) \geq \frac{\delta}{\sqrt{2}}$. So we have $d(a, c)+d(c, b) \geq \frac{\delta}{\sqrt{2}}$. This shows that the $\theta$-metric on $X$ satisfies condition (iii-C) of Theorem 1.2. Thus, by the metrization criterion due to Niemytski and Wilson, we can conclude that, the $\theta$-metric space $X$ is metrizable.

Open question. Can an explicit metric $d$ be constructed with respect to which $b$-metric spaces with coefficient $K>0$ and, respectively $\theta$-metric spaces are metrizable?

Acknowledgements. The authors would like to thank the anonymous reviewers for their comments and suggestions. For the second author, the publication of this article was supported by the 2020 Development Fund of the Babeş-Bolyai University. For the third author this research is funded by the Council of Scientific and Industrial Research (CSIR), Government of India under the Grant Number: 25 (0285)/18/EMR-II. Finally we express our deep gratitude to Professor Pratulananda Das for his valuable suggestions during the preparation of the draft.

\section{REFERENCES}

[1] Aimar, H., Iaffei, B. and Nitti, L., On the Macias-Segovia metrization of quasi-metric spaces, Rev. Un. Mat. Argentina, 41 (1998), No. 2, 67-75

[2] An, V. T., Tuyen, Q. L. and Dung, V. N., Stone-type theorem on b-metric spaces and applications, Topology Appl., 185-186 (2015), 50-64

[3] Bera, A., Garai, H., Damjanovic, B. and Chanda, A. Some interesting results on $\mathcal{F}$-metric spaces, Filomat, 33 (2019), 3257-3268

[4] Berinde, V., Generalized contractions in quasimetric spaces, Seminar on Fixed Point Theory, (1993), 3-9

[5] Boriceanu, M., Petruşel, A. and Rus, I. A., Fixed point theorems for some multivalued generalized contractions in b-metric spaces, International J. Math. and Statistics, 6 (2010), S10, 65-76

[6] Brzdek, J., Karapinar, E. and Petruşel, A., A fixed point theorem and the Ulam stability in generalized dq-metric spaces, J. Math. Anal. Appl., 467 (2018), 501-520

[7] Chittenden, E. W., On the equivalence of Écart and voisinage, Trans. Amer. Math. Soc., 18 (1917), 161-166

[8] Cobzaş, S., b-metric spaces, fixed points and Lipschitz functions, arXiv:1802.02722v3 [math.FA], 25 Mar (2019) 
[9] Czerwik, S., Contraction mappings in b-metric spaces, Acta Math. Univ. Ostraviensis, 1 (1993), 5-11

[10] Czerwik, S., Nonlinear set-valued contraction mappings in b-metric spaces, Atti Semin. Mat. Fis. Univ. Modena, 46 (1998), 263-276

[11] Frink, A. H., Distance functions and the metrization problem, Bull. Amer. Math. Soc., 43 (1937), 133-142

[12] Jleli, M. and Samet, B., On a new generalization of metric spaces, J. Fixed Point Theory Appl., (2018), 20:128

[13] Khamsi, M. A. and Hussain, N., KKM mappings in metric type spaces, Nonlinear Anal., 73 (2010), 3123-3129

[14] Khojasteh, F., Karapinar, E. and Radenovic, S., A-metric space: a generalization, Math. Probl. Eng., 2013 (2013), Article ID 504609

[15] Kirk, W. and Shahzad, N., Fixed Point Theory in Distance Spaces, Cham: Springer 2014

[16] Niemytski, V. W., On the "third axiom of metric space", Trans. Amer. Math. Soc., 29 (1927), 507-513

[17] Rus, I. A., Petruşel, A. and Petruşel, G., Fixed Point Theory, Cluj University Press Cluj-Napoca, 2008

[18] Som, S., Bera, A. and Dey, L. K., Some remarks on the metrizability of $\mathcal{F}$-metric spaces, J. Fixed Point Theory Appl., (2020), 22:17

[19] Wilson, W. A. On semi-metric spaces, Amer. J. Math., 53 (1931), 361-373

${ }^{1}$ DePartment of MATHEMATICS

SCHOOL OF BASIC AND APPLIED SCIENCES

ADAMAS UNIVERSITY, INDIA

Email address: somkakdwip@gmail.com

2 Department of Mathematics

BABEŞ-BOlyai University Cluj-NaPOCA

ROMANIA

${ }^{3}$ ACADEMY OF ROMANIAN SCIENTISTS

BUCHAREST, ROMANIA

Email address: petrusel@math.ubbcluj.ro

${ }^{4}$ Department of Mathematics

NATIONAL INSTITUTE OF TECHNOLOGY DURGAPUR, INDIA.

Email address: lakshmikdey@yahoo.co.in 